\title{
Study of the $\beta$ dependence of confinement and heat transport in ASDEX Upgrade
}

\author{
L. Vermare, F. Ryter, C. Angioni, A.G. Peeters, J. Stober, R. \\ Bilato, L.D. Horton, B. Kurzan, C.F. Maggi, H. Meister, J. \\ Schirmer, G. Tardini and ASDEX Upgrade Team
}

Max-Planck-Institut für Plasmaphysik, EURATOM Association, D-85748 Garching, Germany

\begin{abstract}
First $\beta$ scan experiments have been made in ASDEX Upgrade in H-mode plasmas with type-I ELMs. Two sets of discharges, performed in different density ranges, scan $\beta$ between $\beta_{N}=[1.4-2.2]$ and $\beta_{N}=[1.4-2]$. Global analysis of these dedicated experiments exhibits a strong unfavourable $\beta$ scaling as $B \tau_{t h} \propto \beta^{-0.9}$. This tendency is confirmed by a local analysis which shows an increase of the thermal heat diffusivity with increasing $\beta$ as $\chi_{e f f} / B \propto \beta^{0.65}$. These results are discussed and compared to studies made in DIII-D, JET and JT-60U.
\end{abstract}

\section{Introduction}

Both experimental and theoretical studies show that small scale plasma fluctuations (micro-turbulence), arising from instabilities driven by temperature and density gradients, can generate high levels of heat and particle transport, thus limiting fusion device performance. The understanding of the micro-turbulence induced transport and, consequently, the prediction of the confinement time plays a crucial role in determining the feasibility of a thermonuclear fusion reactor. The analysis of plasma energy transport in terms of dimensionless parameters $[1,2]$ is a powerful technique widely used in order to extrapolate from present day devices to next step experiments, like ITER. In addition 
to this, the experimental scaling of confinement time and local transport as a function of physically relevant quantities can provide useful insight about the nature of the instabilibities responsible for the anomalous transport. This is particularly important for validating the different proposed theories of turbulent transport in tokamak plasmas. Using the scale invariance approach it has been shown [2] that, if the energy transport is dominated by plasma physics (i.e. does not depend on atomic physics) the normalised energy confinement time can be described by a set of dimensionless parameters and be written as :

$$
B \tau_{t h}=F\left(\rho^{*}, \nu^{*}, \beta, q, \epsilon, \delta, \kappa, T_{i} / T_{e}, \ldots\right)
$$

where $B$ is the toroidal magnetic field on axis, $\tau_{t h}$ is the thermal energy confinement time, $\rho^{*}=\rho_{i} / a$ is the normalised ion Larmor radius, $\nu^{*}=\nu_{i} /\left(\epsilon \omega_{b i}\right)$ is the ion collision frequency normalised to the ion bounce frequency $\omega_{b i}=v_{\perp i} / q R(r / 2 R)^{1 / 2}, \beta=2 \mu_{0} p / B^{2}$ is the normalised plasma pressure, $q$ is the safety factor, $\epsilon=a / R$ is the inverse plasma aspect ratio, $\kappa$ is the elongation, $\delta$ is the triangularity, $T_{i}$ and $T_{e}$ are respectively ion and electron temperature.

The present paper focuses on the experimental study of the $\beta$ scaling of transport in the ASDEX Upgrade tokamak. Since the fusion power production $P_{f u s}$ increases with increasing $\beta$, there is large economic interest to operate reactors at as high as possible $\beta$. Furthermore, the knowledge of the $\beta$ dependence of confinement is also important to extrapolate present discharge scenarios to ITER.

In addition, a precise experimental characterisation of the $\beta$ dependence of transport may provide indications about the nature of turbulence. Electrostatic theories, in which finite $\beta$ electromagnetic effects are neglected, predict a weak $\beta$ dependence through geometrical effects like the Shafranov shift. For example, in drift wave theory, transport is expected to slightly decrease with increasing $\beta$ due to the stabilising $\beta$ effect on ITG modes. On the other hand, approaching the ideal $\beta$ limit, electromagnetic mechanisms not only affect electrostatic instabilities but also 
can destabilise electromagnetic modes, generally leading to a strong $\beta$ dependence of turbulent transport $[3,5,4]$.

Experimentally, the $\beta$ dependence of transport is still a subject of controversy: contradictions exist between the prediction of empirical multi-machine scaling laws and the results of dedicated experiments. The widely used empirical scaling law for high confinement mode (H-mode) plasmas with edge localised modes (ELMs), named IPB98 $(y, 2)[7]$, expressed in dimensionless parameters reads :

$$
B \tau_{t h}^{98 y 2} \propto \rho^{*-2.70} \beta^{-0.90} \nu^{*-0.01} q^{-3.0}
$$

This strongly negative $\beta$ dependence suggests that electromagnetic effects may play a role in the turbulent transport process. In agreement with this negative $\beta$ dependence, recent dedicated experiments in JT-60U [8] exhibited a degradation of global confinement time with increasing $\beta$ as $B \tau_{t h} \propto \beta^{-0.6}$. In contrast, dedicated $\beta$ scans performed in DIII-D [9] and JET [10] yielded no $\beta$ dependence, in both global confinement time and local thermal diffusivities.

To contribute to clarifying these discrepancies, first $\beta$ scaling experiments have been performed in ASDEX Upgrade. In the next section, experiments for the ASDEX Upgrade $\beta$ scans are described and the results are presented in section 3. Differences in experimental conditions of $\beta$ scans performed in DIII-D and JET as compared to ASDEX Upgrade and their possible impact on the results are discussed in section 4.

\section{Experimental conditions}

Dedicated $\beta$ scan experiments on ASDEX Upgrade have been performed keeping $\rho^{*}$, $\nu^{*}$ (global and local values), the safety factor $q$ and the plasma shape constant. The parameters $\rho^{*}, \nu^{*}$, as well as the magnetic shear are known to have a strong impact on the plasma core instabilities, while a constant plasma shape is particularly important for the control of the edge behaviour. To vary $\beta$ while keeping $\rho^{*}, \nu^{*}$, the safety factor $q$ and the plasma shape constant, the magnetic field $B$ is varied in these discharges, and 
the main plasma parameters have to satisfy the following relationships:

$$
I \propto B ; n \propto B^{4} ; T \propto B^{2}
$$

Following these requirements, $\beta$ varies as $B^{4}$. As usual, in order to simplify the analysis and to compare with the empirical scaling law, we have made the assumption of considering a power law for the dependence of confinement time on $\beta$ : $B \tau_{t h} \propto$ $\beta^{-\alpha_{\beta}} F\left(\rho^{*}, \nu^{*}, \ldots\right)$. Therefore, the heating power must vary as $P_{h} \propto B^{7+4 \alpha_{\beta}}$. In the following, $\beta$ will correspond to the total normalised pressure including fast particles, the thermal normalised pressure will be written as $\beta_{t h}$ and $\beta_{N} \equiv \beta /(I / a B)$. Experimentally, the $\beta$ range is limited by the $\mathrm{L}-\mathrm{H}$ transition at low $\beta\left(\beta_{N} \approx 1.1\right)$ and by appearance of the Neoclassical Tearing Modes (NTM) at high $\beta\left(\beta_{N} \approx 2.5\right)$. In addition, since the density has to vary as $\beta\left(\propto B^{4}\right)$, another important limitation arises at low $\beta$ from the natural density and at high $\beta$ from the $\mathrm{H} / \mathrm{L}$ transition close to the Greenwald density. In ASDEX Upgrade, the procedure to perform $\beta$ scaling experiments was to first carry out a low $\beta$ discharge at the lowest density reachable and at the lowest power needed to have type-I ELMs. From this discharge, the high $\beta$ discharge was performed trying to reach simultaneously the density and the $\beta$ required to match $\rho^{*}$ and $\nu^{*}$. All these experiments were performed in a single-null divertor plasma configuration and the shape parameters were chosen to closely match the plasma shape of JET and DIII-D experiments $(\delta \simeq 0.2$ and $\kappa \simeq 1.8)$ and $q_{95}=3.8$. The plasma was fuelled by deuterium gas puffing, heated by neutral beam injection (NBI) and in some cases with (H-minority) ICRH heating. The voltage of the NBI injectors was adjusted according to the density to match as well as possible the heating deposition profile between low and high $\beta$ discharges. The divertor cryopump was used to control the plasma density in low density discharges and turned off to help reaching high density. The density profiles are calculated from interferometer and lithium beam data. The electron temperature profiles are measured by the Thomson scattering diagnostic. The charge exchange recombination spectroscopy (CXRS) during neutral beam injection provides a measure of the ion temperature and 
rotation profiles. Equilibria and q-profiles are reconstructed using the MHD equilibrium code CLISTE [12]. The heating deposition profiles for NBI and ICRH are calculated respectively by the Monte-Carlo code FAFNER [13] and the TORIC code [14]. The power balance analyses have been made using the ASTRA code [15] and comparisons carried out with the TRANSP code [16].

\section{Experimental results}

In the study presented here, two separate $\beta$ scans in H-mode with Type-I ELMs have been performed in ASDEX Upgrade. The $\beta$ ranges are $\beta_{N}=[1.4-2.2]$ (scan A) and $\beta_{N}=[1.4-2]$ (scan B) and cover two different density windows. The heating power was feedback-controlled to obtain a stationary phase at the required $\beta$. Engineering parameters as well as global dimensionless quantities of both scans are summarised in table 1. Global parameter calculations and analyses were performed over a stationary phase characterised by a time window width $\Delta t$. For discharges 21225 and $21226 \Delta t$ is comparable to the confinement time. However, during these discharges the time window was chosen at the maximum of $\beta$ which correspond also to the maximum of the confinement time. Therefore, enlarging the time window leads to a stronger $\beta$ degradation. The $H$ factors of these discharges are close to 1 showing that the thermal confinement times are consistent with the IPB98(y,2) scaling law. Considering only "random" errors, the uncertainties on global parameters for both scans are estimated as $3.1 \%$ for $\rho^{*}, 15.4 \%$ for $\nu^{*}, 3.2 \%$ for $\beta$ and $9 \%$ for $B \tau_{t h}$. Global (deduced from stored energy) and local quantities such as $\rho^{*}, \nu^{*}$ and plasma shape parameters have to be well matched between discharges of the same scan in order to separate the $\beta$ dependence of $B \tau_{t h}$ from the dependences induced by these parameters.

The first scan composed of one low $\beta$ and three high $\beta$ discharges was performed at high density, $\bar{n}_{e_{19}}=[7.2-9.4] \mathrm{m}^{-3}$, which corresponds in term of Greenwald fraction, $F_{G W}$, to $F_{G W}=[57 \%-68 \%]$. In this scan, in addition to NBI heating, ICRH was used in an attempt to adjust the heating profile deposition. Even without cryopump, it was 
difficult to reach the density required $\left(\bar{n}_{e_{19}}=9.7\right)$ while keeping $\beta_{N}=2$. Thus, in the scan A, there is a significant $\nu^{*}$ and $\rho^{*}$ mismatch while $q_{95}, \kappa$ and $\delta$ were well matched.

The scan B, composed of two discharges, was carried out at lower density than the $\operatorname{scan} \mathrm{A}, \bar{n}_{e_{19}}=[5.3-8] \mathrm{m}^{-3}$, or in term of Greenwald fraction, $F_{G W}=[43 \%-57 \%]$. Both discharges were performed with NBI heating only. Matching of global parameters (within $15 \%$ for $\nu^{*}$ and within $2 \%$ for $\rho^{*}$ which are within the error-bars) as well as heating deposition profiles are clearly better than in the scan A.

\subsection{Global confinement time analysis}

Figure 1 shows the normalised thermal energy confinement time $B \tau_{t h}$ as a function of $\beta_{N}$ for the six previously described discharges. For each scan, there is a clear degradation of the global confinement with increasing $\beta$. Linear regressions of each pair of discharges yield a $\beta$ dependence of $B \tau_{t h}$ which varies significantly from $\alpha_{\beta}=0.6 \pm 0.4$ to $\alpha_{\beta}=1.8 \pm 0.7$ (table 2). When all discharges of scan A are considered, $\alpha_{\beta}=1.16$, while scan B (red) gives $B \tau_{t h} \propto \beta_{t h}^{-1.2 \pm 0.6}$. Although uncertainties on $\alpha_{\beta}$ are quite large , in particular when regression is made on only 2 points, $\alpha_{\beta}$ remains always positive within the error-bars. Note that the uncertainties reported in table 2 were calculated taking into account only the uncertainties on $\tau_{t h}$ and $\beta_{t h}$. The inclusion of $\rho^{*}$ and $\nu^{*}$ uncertainties $\left(\Delta \rho^{*}\right.$ and $\left.\Delta \nu^{*}\right)$ requires assumptions about the dependence of the global confinement time on $\rho^{*}$ and $\nu^{*}$. The Gyro-Bohm scaling $B \tau_{t h} \propto \rho^{*-3}$ is well established in Hmode plasmas and has been verified on different tokamaks $[17,18]$. The $\nu^{*}$ dependence in dedicated experiments was found to be weak, $\nu^{*-0.3}$, at low density $[18,19]$ and stronger, $\nu^{*-1}$, at higher density [20]. Since $\Delta \rho^{*}$ and $\Delta \nu^{*}$ are correlated via the stored energy and density uncertainties, $\Delta \alpha_{\beta}$ must be calculated using directly $\Delta W$ and $\Delta n$. For example, assuming $B \tau_{t h} \propto \rho^{*-3} \nu^{*-1}$ for the pair 21403-21426, $\Delta \alpha_{\beta}=0.9$. On the contrary, assuming $B \tau_{t h} \propto \rho^{*-3} \nu^{*-0.3}$, the uncertainty on $\alpha_{\beta}$ almost does not change as compared to the values reported on table 2 .

As mentioned before, the $\rho^{*}$ and $\nu^{*}$ matching between low and high $\beta$ discharges 
is not perfect and differs significantly for each pair of discharge. Therefore, parameter mismatching affects in a different way each pair of discharges and may explain the large scattering of $\alpha_{\beta}$ values obtained. In order to compensate for these effects, the global confinement time can be corrected using its $\rho^{*}$ and $\nu^{*}$ dependence obtained from previous experiments.

Corrections considering the dependences described above have been applied, separately and together, to each pair of discharges and the resulting $\beta$ dependences are summarised in table 2. The $\rho^{*}$ correction tends to decrease the $\beta$ dependence for both scans. In contrast, the $\nu^{*}$ correction acts in opposite directions in scan A and scan B. In the first scan, the $\nu^{*}$ mismatch leads to an underestimate of $\alpha_{\beta}$ while it leads to an overestimate in scan B. Note that applying no $\nu^{*}$ correction and only the Gyro-Bohm scaling correspond almost to the dimensionless scaling obtained from the IPB98(y,2).

For the first scan, the significant $\rho^{*}$ and $\nu^{*}$ mismatches induce a strong influence of corrections and in particular of the choice of the $\nu^{*}$ correction. However, applying the corrections, the $\beta$ scaling remains clearly unfavourable in both scans.

Considering the $\nu^{*}$ correction for high density, scan A leads to $B \tau_{t h} \propto \beta^{*-1.38}$ considering all discharges, while $\alpha_{\beta}=1.08$ for the pair 20989-21101. The $\beta$ dependence obtained for discharges 21225 and 21226 is clearly stronger (up to $\alpha_{\beta}=1.93$ ). As mentioned above, in scan A, ICRH has been added to NBI. Applying ICRH has been shown to flatten the density profile [21], effect which increases with $P_{I C R H}$. Therefore, in shots 21225 and 21226 , the high ICRH power of $3.8 \mathrm{MW}$ induced a stronger flattening of the density profile than in shots 21101 and 20889 with much lower ICRH powers. Consequently, in discharges 21225 and 21226 much stronger gas puffing was required to reach the desired line averaged density than in 21101. This produces an extremely flat density profile with very high pedestal density, inducing low pedestal ion temperature. In addition, the ELMs frequency is much higher $180 \mathrm{~Hz}$ compared to $90 \mathrm{~Hz}$ in 20989 and 21101.

For scan B, applying the low density correction $\left(B \tau_{t h} \propto \nu^{*-0.3}\right)$ yields $B \tau_{t h} \propto \beta_{t h}^{-0.94}$ 
which agrees with the result from scan A (pair 20989-21101) using high density $\nu^{*}$ correction. The latter result are in accordance with the $\beta$ dependence deduced from the IPB98 $(\mathrm{y}, 2)[7]$ and the unfavourable $\beta$ scaling found recently in JT-60U [8]. In order to cancel completely the $\beta$ degradation in scan $\mathrm{B}$, considering a Gyro-Bohm scaling, a $\nu^{*}$ dependence as $B \tau \propto \nu^{*-2}$ would be required. However, such $\nu^{*}$ dependence has never been found in $\nu^{*}$ scaling experiments or in empirical scaling laws.

\subsection{Local heat transport analysis}

In addition to matching the global normalised parameters, it is also important to verify that of local dimensionless quantities which are expected to play a role in heat transport. The local study of transport was performed on the best matched scan (scan B). All radial profiles presented in the following are plotted as a function of the normalised toroidal flux coordinate $\rho_{t}$. First of all, the heating deposition profiles normalised to $B^{7}$ (if $\alpha_{\beta}=0$, $P_{h} \propto B^{7}$ ) and plotted in figure 2 a show that, in order to reach the $\beta$ required, more additional power was needed than expected without $\beta$ degradation. This behaviour agrees with the negative dependence found in the global analysis. Considering $\alpha_{\beta}=1$, the profiles $P_{h} / B^{7+4 \alpha_{\beta}}$ are correctly matched as we can see in figure $2 \mathrm{~b}$. The radial profiles of the kinetic normalised plasma pressure $\beta_{k i n}$ and of the quantity $\beta_{k i n} / B^{4}$ are shown in figure 3 . The profile of the quantity $\beta_{k i n} / B^{4}$ are similar for low and high $\beta$ discharges even at $\rho_{t}=0.85$. The H-mode edge pedestal and the core seem to vary in concert but in these discharges, no accurate edge measurements were available. For this reason, in the following, all radial profiles will be plotted between $\rho_{t}=[0-0.85]$ and no detailed analysis of the plasma edge will be done. On the profiles of $\beta_{k i n} / B^{4}$, only around $\rho_{t}=0.5$ there is a small gap, the high $\beta$ discharge being slightly higher, but the difference is within the uncertainties.

Density and temperature profiles show that the normalised gradient lengths $R / L_{n}$ and $R / L_{T}$ (for ions and electrons) do not change significatively between low and high $\beta$ discharges. This feature suggests that core transport is governed by microinstabilities 
like ITG and TEMs, with consequent stiff behaviour of the temperature profiles in response to variations of the heat fluxes. Therefore, in these conditions the heat conductivity in the core might be strongly affected by the confinement at the plasma edge.

Figure 4 shows the profiles of the other relevant dimensionless quantities $\rho^{*}, \nu^{*}$, $T_{i} / T_{e}, \mathrm{q}$, effective ion charge $Z_{\text {eff }}, v_{\text {rot }} /\left(T_{i} / m_{i}\right)^{1 / 2}$ and $R \nabla v_{\text {rot }} /\left(T_{i} / m_{i}\right)^{1 / 2}$. These profiles are well matched between the low and high $\beta$ discharges except for $Z_{\text {eff }}$. The mismatch does not exceed $5 \%$ for $\rho^{*}$ and $20 \%$ for $\nu^{*}$ profiles. The small difference in the safety factor profiles at the center is not really relevant because the uncertainties are quite large at this location due to the sawtooth activity. The ratio $T_{i} / T_{e}$ varies radially, the ion temperature is higher than electron temperature at the edge and the mismatch between the two discharges reaches at most $15 \%$. Note that higher values of $T_{i} / T_{e}$ are expected to stabilise electrostatic modes ITG [22].

The level of impurity is higher in the low $\beta$ discharge for which $Z_{\text {eff }}$ is around 2 while $Z_{\text {eff }} \simeq 1.5$ during the high $\beta$ discharge. This general feature that $Z_{\text {eff }}$ decreases with increasing density is commonly observed in ASDEX Upgrade ELMy H-mode plasmas [23]. In the local analysis, the effect of the dilution is taken into account computing the power balance using the experimental $Z_{\text {eff }}$ profiles. Calculations have been made considering as main impurity Carbon, Helium or Bore. The relative behaviour of the thermal conductivity profiles (comparison of low and high $\beta$ discharges) does not change from one impurity to another.

From theoretical standpoint, $Z_{\text {eff }}$ affects the stability of ITG modes by ion dilution, such that increasing $Z_{\text {eff }}$ has a stabilizing effect on ITG, stronger for light impurities than for heavy impurities $[24,25]$. Therefore, the lower value of $Z_{\text {eff }}$ in the high $\beta$ discharge could amplify the $\beta$ degradation. To estimate the $Z_{\text {eff }}$ mismatch effect, we performed linear gyrokinetic simulations in the experimental conditions (GS2 code $[26,27])$. They show that a change in $Z_{\text {eff }}$ from 2 to 1.5 leads to an increase of the ITG growth rate of around $5 \%$ and a shift in the normalised gradient length $\left(R / L_{T}\right)$ 
threshold of less than 0.5 . Thus, the mismatch in $Z_{\text {eff }}$ is expected to only play a minor role in the confinement and heat transport of the discussed experiments.

The rotational shear has a stabilising effect on transport, therefore the relative influence of the toroidal rotational velocity $v_{\text {rot }}$, must be kept constant during dimensionless scaling. Considering that $v_{r o t}$ is the dominant term in the radial electric field $\left(E_{r} \approx B_{p} v_{r o t}\right)$, the shearing rate may be written as $\omega_{E \times B} \approx \epsilon / q \nabla v_{\text {rot }}$. To compare with previous experiments, the profiles of the normalised velocity $v_{\text {rot }} /\left(T_{i} / m_{i}\right)^{1 / 2}$ is plotted in figure 4. The radial profiles of this quantity which is equivalent to the Mach number $\left(\propto\left(T_{e} / T_{i}\right)^{1 / 2}\right)$, are well matched between low and high $\beta$ discharge. The normalised toroidal velocity is slightly higher in the high $\beta$ discharge but the mismatch do not exceed $5 \%$ even at $\rho_{t}=0.8$. However, as the $E \times B$ shearing rate is proportional to the gradient of the toroidal rotation velocity, the quantity $R \nabla v_{\text {rot }} /\left(T_{i} / m_{i}\right)^{1 / 2}$ plotted in figure 4 must be also checked. The latter shows that the behavior of this quantity is in opposite direction than the normalised $v_{\text {rot }}$. For the low $\beta$ discharge, $R \nabla v_{\text {rot }} /\left(T_{i} / m_{i}\right)^{1 / 2}$ is slightly higher (6\%) than in the high $\beta$ discharge. This mismatch is very small but means that the $E \times B$ stabilization is larger in the lower $\beta$. A recent experimental study on the role of $T_{i} / T_{e}$ and $\nabla v_{\text {rot }}$ on ion heat transport in ASDEX Upgrade H-modes has presented the ITG threshold $\left(R / L_{T_{i}}\right)$ dependence on $R \nabla v_{\text {rot }} /\left(T_{i} / m_{i}\right)^{1 / 2}$ [28]. In this reference it is shown that decreasing the quantity $R \nabla v_{\text {rot }} /\left(T_{i} / m_{i}\right)^{1 / 2}$ by $6 \%$ at fixed ratio $T_{i} / T_{e}$ has a very weak effect on $R / L_{T_{i}}$ and leads to a decrease of $R / L_{T_{i}}$ smaller than $5 \%$. Finally, this effect which affects only the ion channel may increase only very slightly the effective local transport.

To evaluate the $\beta$ dependence of the local heat transport, experimental thermal diffusivities have been calculated from the radial power balance analysis. The heat flux has been assumed to be purely diffusive, therefore the thermal diffusivity is given by $\chi=-q / n \nabla T$. In the following, the effective heat diffusivity is defined by :

$$
\chi_{e f f}=\frac{q_{e}+q_{i}}{n_{e} \nabla T_{e}+n_{i} \nabla T_{i}}
$$

where $q_{e}$ and $q_{i}$ are respectively, the electron and ion heat flux. 
The total stored energy obtained by the equilibrium reconstruction and by the sum of kinetic energy from the experimental profiles and fast particle energy (from FAFNER) agrees within 5\%. For comparison, the power balance calculations deduced using the TRANSP code yielded a similar $\beta$ dependence of the thermal conductivity.

The normalised $\chi_{e f f} / \chi_{B} \propto \chi_{e f f} / B$ profiles, where $\chi_{B}$ is the Bohm diffusion coefficient, are plotted in figure 5. The error-bars reported in figure 5 were calculated as follows. First, for each of the two discharges of scan B, a set of $N=500$ fitted profiles $\left(T_{e}, T_{i}\right.$ and $\left.n_{e}\right)$ has been generated by varying randomly (using a Gaussian distribution) the experimental points within their experimental errors. The latter were taken as $15 \%$ for $T_{e}, 10 \%$ for $T_{i}$ and $5 \%$ for $n_{e}$. Note that in the heat diffusivity, the largest uncertainties come from the temperature gradients. Secondly, for each set of the 500 profiles, a $\chi_{\text {eff }}$ profile was calculated by power balance. In this procedure, the heating deposition profiles are kept the same and have not been re-computed using FAFNER on the new set of profiles. However, FAFNER and ASTRA calculations show that varying density and temperature profiles in their error-bars lead at most to a change in $\chi_{e f f}$ around $5 \%$. The distribution of $\chi_{e f f}$ obtained in this way is not symmetric. For this reason, the $\chi_{e f f} / B$ plotted in figure 5 is taken at the maximum of the distribution function and the error-bars are the half-widths of each sides of this maximum. Following this procedure, the uncertainties are found to be around $15 \%$ at mid-radius and larger at the edge. The effective diffusivity is clearly higher for the high $\beta$ discharge on a large radial area in the confinement zone $\left(\rho_{t}=0.3-0.9\right)$. For these profiles, the parameter $\alpha_{\beta}$, defined as $\chi_{e f f} / B \propto \beta^{\alpha_{\beta}}$, is plotted in figure 6 .

The average $\beta$ dependence of $\chi_{e f f} / B$ from $\rho_{t}=0.2$ to $\rho_{t}=0.8$ corresponds to $\alpha_{\beta}=0.65$. The $\beta$ dependence changes significatively with radius from $\alpha_{\beta} \simeq 0$ at $\rho_{t}=0.2$ to $\alpha_{\beta} \simeq 1$ between $\rho_{t}=[0.5-0.7]$. 


\section{Conclusion}

First dedicated experiments on $\beta$ scaling in ASDEX-Upgrade show a clearly unfavourable $\beta$ dependence of transport in both global and local analyses. The global confinement time is found to vary as $B \tau_{t h} \propto \beta^{-1.2 \pm 0.6}$. Since in these experiments the $\rho^{*}$ and $\nu^{*}$ profiles are not perfectly matched, attempts to correct these mismatches have been made. These corrections did not cancel the strong negative $\beta$ degradation and lead to a $\beta$ dependence as $B \tau_{t h} \propto \beta^{-0.9}$. In addition, the local effective heat diffusivity is enhanced with increasing $\beta$ as $\chi_{\text {eff }} \propto \beta^{0.65}$ (on average from $\rho_{t}=0.2$ to $\rho_{t}=0.8$ ). These results are in agreement with the $\beta$ exponents derived from the ITER-IPB98 $(\mathrm{y}, 2)$ scaling law and with the results of JT-60U dedicated experiments. However, they do not agree with DIII-D and JET experiments, in which no $\beta$ dependence has been found. Although the experimental conditions of $\beta$ scans performed in JET, DIII-D and ASDEX Upgrade are very similar, two main differences can be identified. The possible reasons for these differences are discussed in the following.

Firstly, in ASDEX Upgrade, the collisionality is higher at $\rho_{t}=0.5$ by a factor 5 as compared to JET and DIII-D experiments while the Greenwald fractions are in similar ranges (from $26 \%$ for the low $\beta$ to $68 \%$ for the higher $\beta$ ). According to the present results from dedicated experiments on the $\nu^{*}$ dependence of confinement time $[18,19,20]$, the effect of collisionality via the $\nu^{*}$ mismatch cannot be responsible for the apparent $\beta$ degradation, as it was shown in section 2.1. However, collisionality may play a role in the $\beta$ scaling by changing the turbulence regime.

Secondly, a comparison of the plasma shapes in these $\beta$ scans indicates that the elongation is the same in DIII-D and AUG $(\kappa \simeq 1.8)$ while $\kappa=1.6$ in JET experiments. The average triangularity is equal in JET and ASDEX Upgrade $(\delta=0.2)$ and slightly higher in DIII-D $(\delta \simeq 0.3)$, but the upper triangularity $\delta_{u p}$ is clearly smaller in AUG $\delta_{u p}^{A U G} \simeq 0.06$ as compared to both DIII-D and $\operatorname{JET}\left(\delta_{u p}^{D I I I-D} \simeq \delta_{u p}^{J E T} \simeq 0.2\right)$ plasmas. The plasma shape is known to influence ELMs and H-mode pedestal properties. In particular, high triangularity shapes exhibit an increased edge pressure gradient and lead 
to a better confinement $[32,33,34,35]$. In addition, at high $\delta$, confinement degradation with increasing density occurs at higher density as compared to low $\delta$ plasmas [36]. These two effects can be linked to the observed $\beta$ degradation in ASDEX Upgrade. As suggested in [37], the upper triangularity might play a role in the $\beta$ dependence of transport. In this reference, from analyzing an ASDEX Upgrade subset of the ITPA (International Tokamak Physics Activity) confinement database, a $\beta$ degradation has been found and attributed to $\delta_{u p}<0$ plasmas. Although in the experiments presented here $\delta_{u p}>0$, the tendency is in the same direction.

The influence of the plasma shape suggest that the $\beta$ dependence of confinement is a plasma edge effect. In contrast, the increase of the normalised thermal conductivity $\chi_{e f f} / B$ with increasing $\beta$ is not only localized at the edge but it is also visible at midradius. However, in the confinement region, density and temperature gradient lengths do not change significatively between low and high $\beta$ discharge. In these conditions, a strongly negative $\beta$ dependence of the edge plasma may be sufficient to explain the increase of the thermal conductivity at mid-radius.

To investigate the role of the edge in the $\beta$ scaling, new $\beta$ scans with changing the plasma shape with edge measurements are planned in ASDEX Upgrade.

\section{Acknowledgments}

The authors would like to acknowledge the valuable support of the whole AUG Team. One of the authors (L.V.) is grateful to A. Bottino for helpful suggestions and valuable discussions.

L.V. acknowledges financial support for this work from the EURATOM programme of the European Community in the form of a Individual Fellowship, Contract No FU06028309 . 


\section{Figures}

\begin{tabular}{|c|c|c|c|c|c|c|}
\hline \multirow[b]{2}{*}{ pulse } & \multicolumn{4}{|c|}{ scan A } & \multicolumn{2}{|c|}{$\operatorname{scan} B$} \\
\hline & 20989 & 21101 & 21225 & 21226 & 21403 & 21426 \\
\hline$\Delta t(\mathrm{~ms})$ & 2000 & 300 & 100 & 100 & 600 & 400 \\
\hline$\tau_{t h}(m s)$ & 135.5 & 94.81 & 67.86 & 60.67 & 160.9 & 89.47 \\
\hline $\mathrm{B}(\mathrm{T})$ & -2.10 & -2.29 & -2.28 & -2.28 & -2.12 & -2.33 \\
\hline I(MA) & 1.00 & 1.10 & 1.09 & 1.09 & 0.99 & 1.09 \\
\hline $\bar{n}_{e}\left(10^{19} m^{-3}\right)$ & 7.2 & 8.6 & 8.7 & 9.7 & 5.3 & 8 \\
\hline $\bar{n}_{e} / B^{4}\left(10^{19} m^{-3} / T^{4}\right)$ & 0.37 & 0.31 & 0.32 & 0.36 & 0.26 & 0.27 \\
\hline$F_{G W}(\%)$ & 57 & 64 & 63 & 68 & 43 & 54 \\
\hline$P_{N B I}(M W)$ & 2.4 & 7.75 & 7.5 & 10 & 2.6 & 8.75 \\
\hline$P_{I C R H}(\mathrm{MW})$ & 1.11 & 0.6 & 3.8 & 3.8 & 0 & 0 \\
\hline$P_{t o t} / B^{7}\left(M W / T^{7}\right)$ & 0.019 & 0.025 & 0.035 & 0.043 & 0.013 & 0.023 \\
\hline$q_{95}$ & 3.8 & 3.9 & 3.8 & 3.8 & 3.8 & 3.8 \\
\hline $\mathrm{a} / \mathrm{R}$ & 0.30 & 0.31 & 0.30 & 0.30 & 0.30 & 0.30 \\
\hline$\kappa$ & 1.84 & 1.79 & 1.84 & 1.83 & 1.82 & 1.86 \\
\hline$\delta$ & 0.21 & 0.24 & 0.22 & 0.23 & 0.20 & 0.22 \\
\hline$\rho^{*}$ & 0.0061 & 0.0069 & 0.0066 & 0.0067 & 0.0070 & 0.0071 \\
\hline$\nu^{*}$ & 0.610 & 0.300 & 0.396 & 0.388 & 0.243 & 0.279 \\
\hline$\beta_{N}$ & 1.41 & 2.22 & 1.97 & 2.22 & 1.41 & 2.05 \\
\hline$\beta_{t h}$ & 1.32 & 2.07 & 1.87 & 2.09 & 1.30 & 1.93 \\
\hline$B \tau_{t h}$ & 0.284 & 0.217 & 0.155 & 0.138 & 0.342 & 0.208 \\
\hline$H_{I P B 98(y, 2)}$ & 0.99 & 1.11 & 0.89 & 0.90 & 1.16 & 1.01 \\
\hline
\end{tabular}

Table 1. Engineering and dimensionless parameters for the two $\beta$ scans. Parameters are averaged over time windows with a width $\Delta t$. 


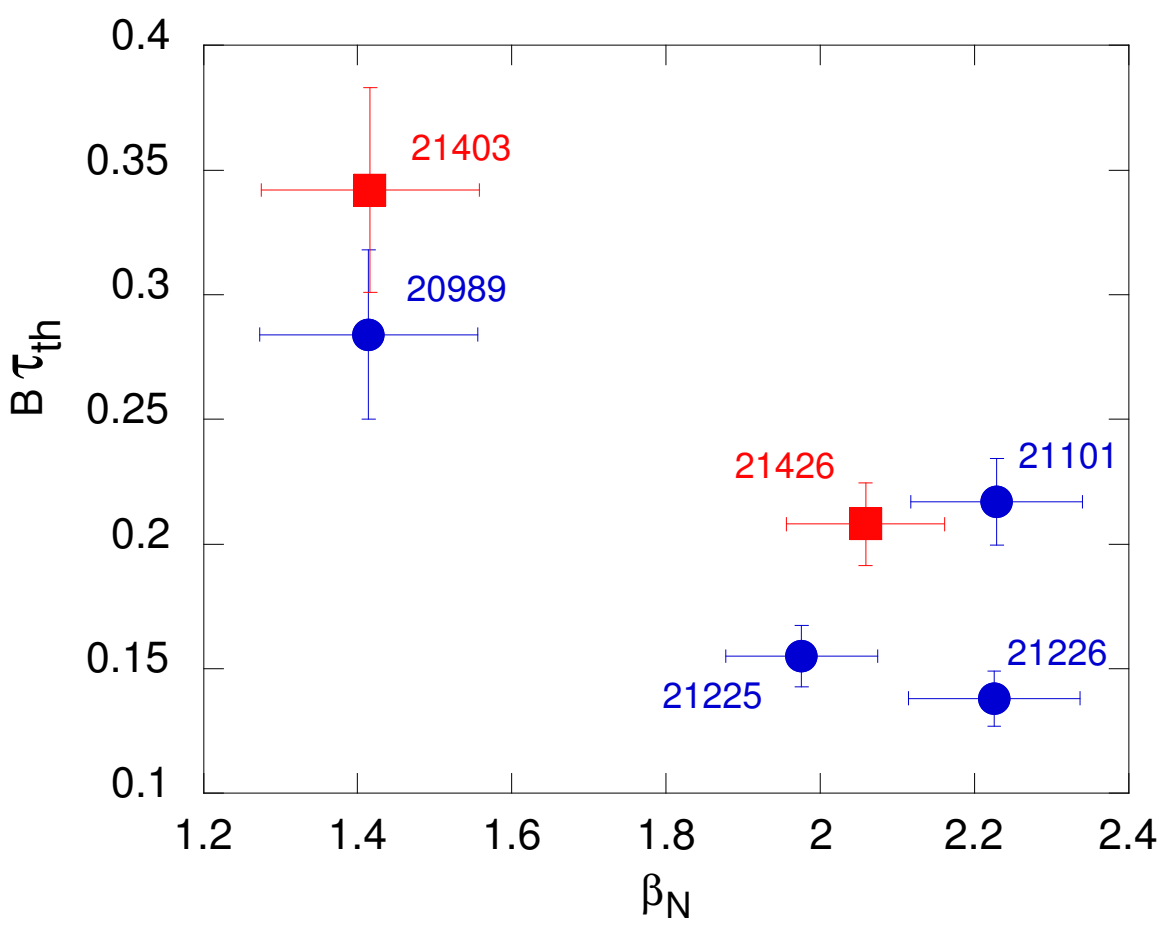

Figure 1. Normalized thermal energy confinement time as a function of $\beta_{N}$ for the discharges given in Table 1 . 


\begin{tabular}{|l|cccc|}
\hline & $20989-21101$ & $20989-21225$ & $20989-21226$ & $21403-21426$ \\
\hline$\alpha_{\beta}$ & $0.6 \pm 0.4$ & $1.8 \pm 0.7$ & $1.6 \pm 0.5$ & $1.2 \pm 0.6$ \\
\hline \multicolumn{4}{|l|}{ with corrections } \\
\hline$\rho^{*-3}$ & -0.18 & 1.09 & 0.94 & 1.14 \\
$\nu^{*-0.3}$ & 0.98 & 2.02 & 1.75 & 1.08 \\
$\nu^{*-1}$ & 1.86 & 2.61 & 2.18 & 0.69 \\
$\rho^{*-3} * \nu^{*-0.3}$ & 0.26 & 1.16 & 1.13 & 0.94 \\
$\rho^{*-3} * \nu^{*-1}$ & 1.08 & 1.93 & 1.56 & 0.58 \\
\hline
\end{tabular}

Table 2. $\beta$ dependence obtained from linear regressions performed on each pair of discharges listed in Table 1 with and without mismatch corrections. 

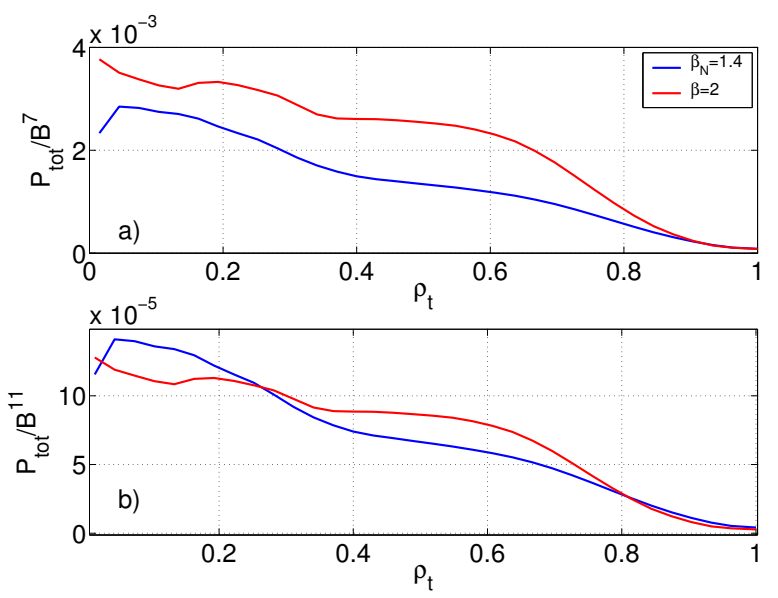

Figure 2. a) Radial profiles of the heating power deposition normalized to $B^{7}$ as a function of the normalized toroidal flux coordinate for discharges $21403\left(\beta_{N}=1.4\right)$ and $21426\left(\beta_{N}=2\right)$. b) Profiles of the heating power deposition normalized to $B^{7+4 \alpha_{\beta}}$ with $\alpha_{b e t a}=1$ 

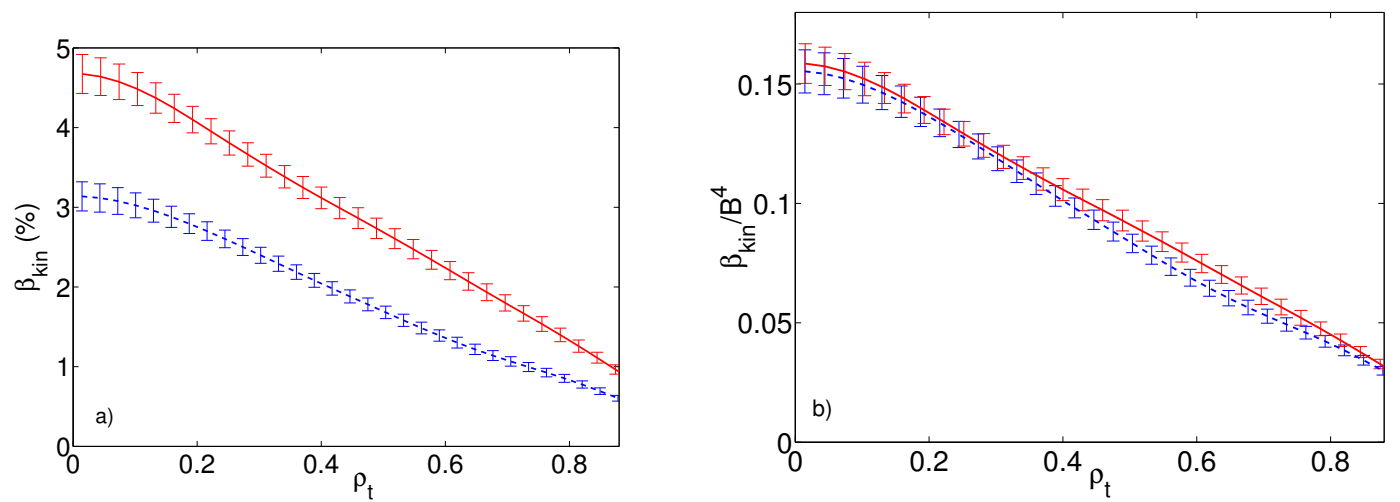

Figure 3. a) Radial profiles of thermal normalized pressure $\beta_{t h}$ for discharges 21403 and 21426. b) Radial profiles of $\beta_{t h}$ normalized to $B^{4}$ for discharges 21403 and 21426. 

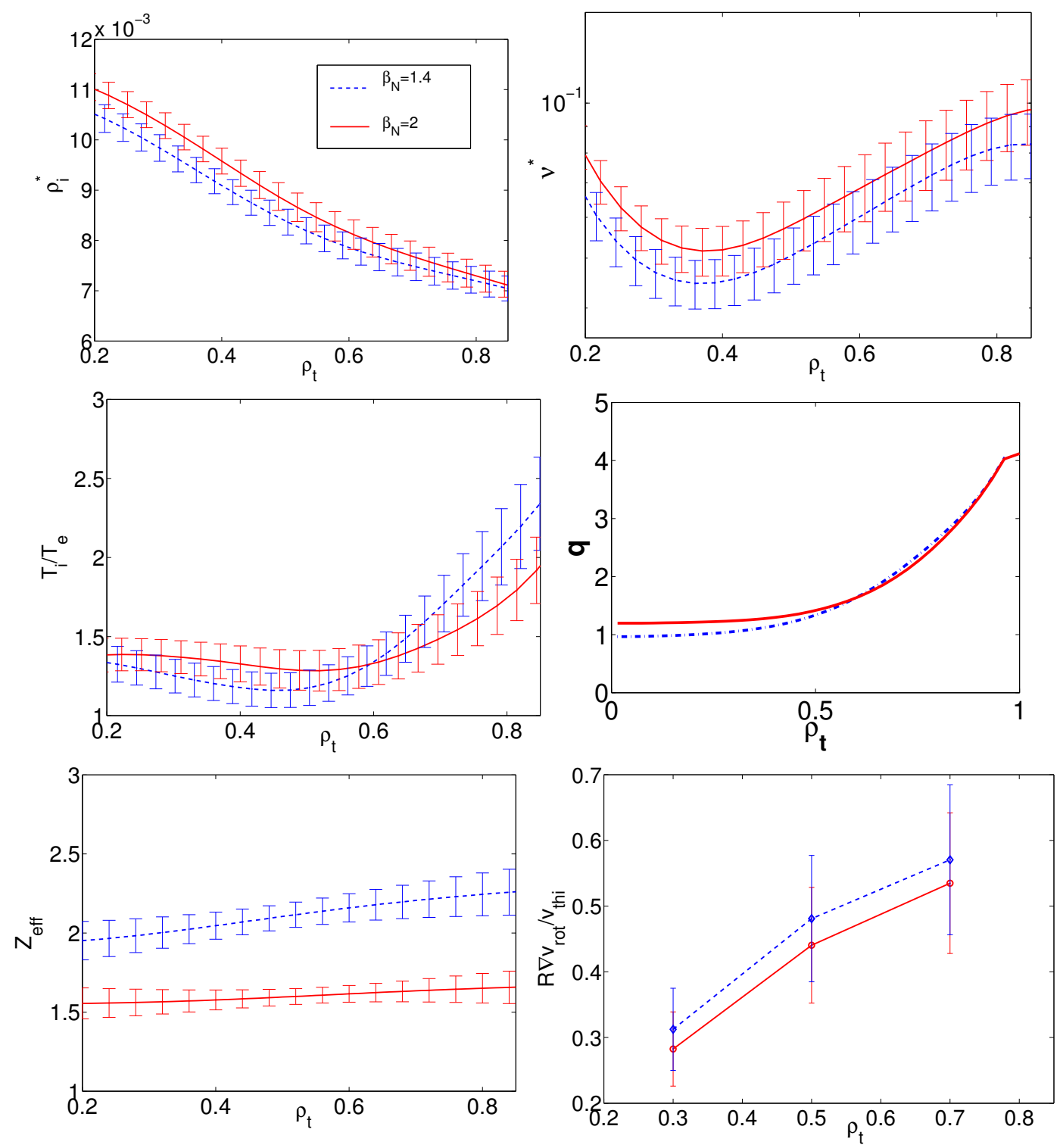

Figure 4. Radial profiles of $\rho^{*}, \nu^{*}, T_{i} / T_{e}$, safety factor $q, Z_{\text {eff }}$ and $R \nabla v_{r o t} / v_{t h i}$ for discharges 21403 and 21426. 


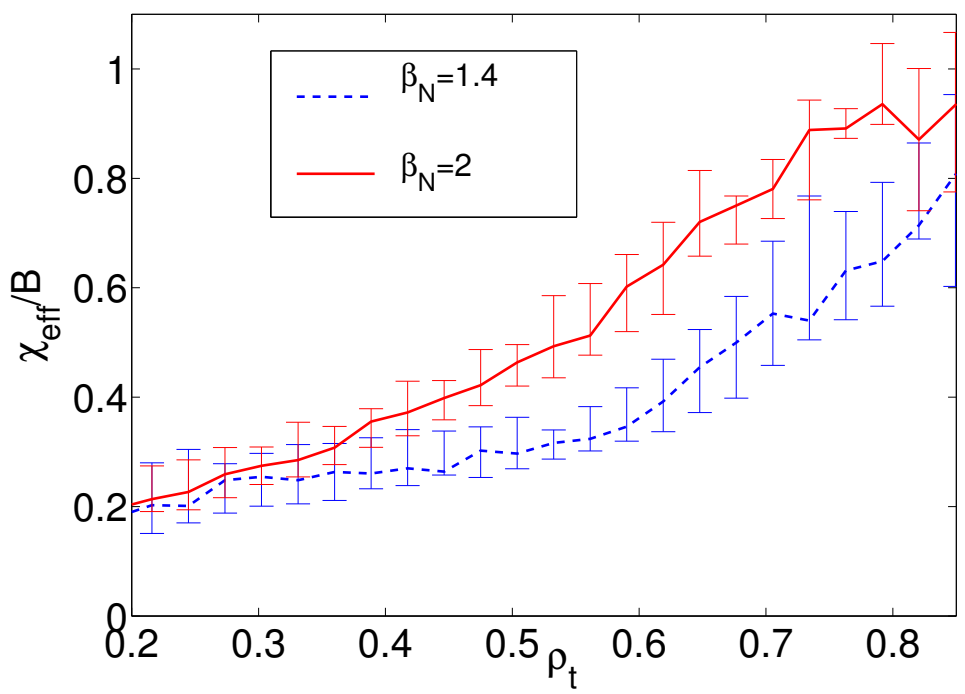

Figure 5. Radial profiles of the effective thermal diffusivity normalized to $B$ for discharges 21403 and 21426. 


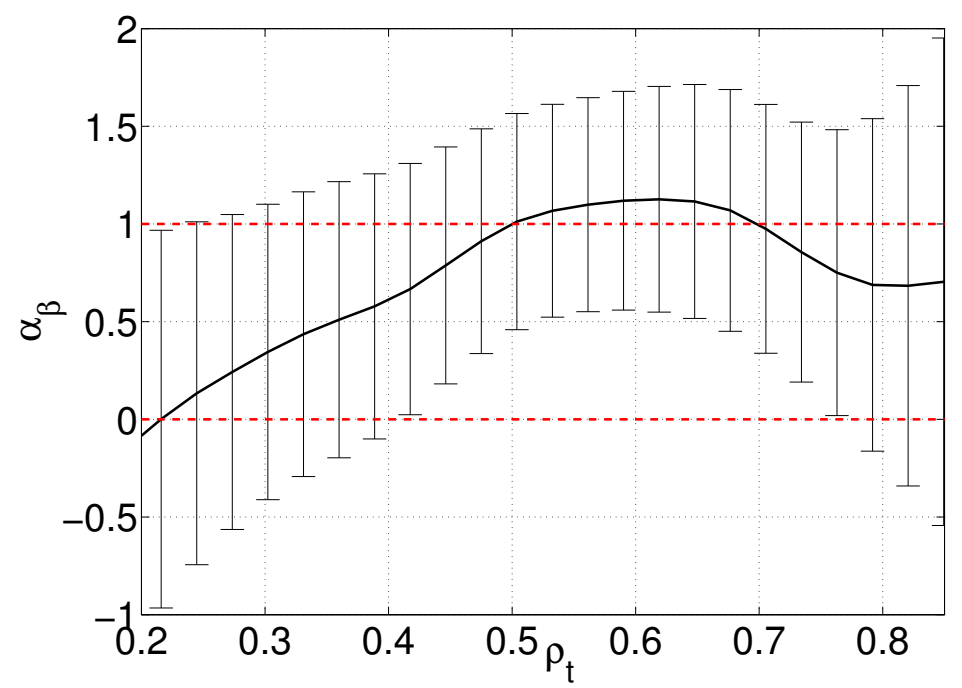

Figure 6. Radial profiles of $\alpha_{\beta}$ from $\chi_{e f f} / B$ for discharges 21403 and 21426. 


\section{References}

[1] B.B. Kadomtsev, Sov. J. Plasma Phys., Vol. 1, No. 4, (1975)

[2] J.W. Connor and J.B. Taylor, Nuclear Fusion, Vol. 17, No. 5, 1047 (1977)

[3] B. Snyder and G. W. Hammett, Phys. Plasmas 8, 744 (2001)

[4] J. Candy, Phys. Plasmas 12, 072307-1/072307-8 (2005)

[5] G. L. Falchetto, J. Vaclavik, and L. Villard, Phys. Plasmas 10, 1424 (2003)

[6] C. Bourdelle et al. Phys. Plasmas 10, 2881 (2003)

[7] ITER Physics Expert Group on Confinement and Transport and Confinement Modelling and Database, et al., Nuclear Fusion 39, 2175 (1999)

[8] H. Urano, T. Takizuka et al., Nuclear Fusion, Vol. 46, No. 8, 781-787 (2006)

[9] C.C. Petty, T.C. Luce, D.C. McDonald et al., Physics of Plasma 11, 5 (2004)

[10] D.C. McDonald et al., Plasma Phy. and Control. Fusion 46, A-215-A-225 (2004)

[11] V. Mertens et al. , Nuclear Fusion, Vol. 37, No. 11, 1607-1614 (1997)

[12] P.J.Mc Carthy et al., 27th EPS Conf. on Contr. Fusion and Plasma Physics, Budapest Vol. 24B, P-440 (2000).

[13] G. G. Lister, "FAFNER - A Fully 3-D Neutral Beam Injection Code Using Monte Carlo Methods", IPP-report, 4-222 (1985)

[14] M. Brambilla and R. Bilato, Nuclear Fusion, 46, No. 7 , S387-S396 (2006)

[15] G.V. Pereverzev and P.N. Yushmanov, IPP report 5/98, February 2002

[16] A. Pankin, D. McCune, R. Andre etal., Comp. Phys. Comm 159, No. 3, 157 (2004)

[17] C.C. Petty, T.C. Luce, K. H. Burrell et al., Physics of Plasma 2, 2342 (1995)

[18] JET Team (presented by J.G. Cordey) in Fusion Energy 1996 (Proc. 16th Int. Conf. Montreal, 1996), Vol., p.611, IAEA, Vienna (1997)

[19] H. Shirai, T. Takizuka, Y, Koide et al., Plasma Phy. and Control. Fusion 42, 1193 (2000)

[20] M. Greenwald et al., Plasma Phy. and Control. Fusion 40, 789-792 (1998)

[21] J. Stober et al., Plasma Phy. and Control. Fusion 43, A39-A53 (2001)

[22] X. Q. Xu and M.N. Rosenbluth, Phys. Fluids B 3 (1991)

[23] H. Meister, L.D. Horton, B. Kurzan et al., 30th EPS Conf. on Contr. Fusion and Plasma Physics, St. Petersburg Vol. 27A, P-1.136 (2003)

[24] R.R. Dominguez and M.N. Rosenbluth, Nuclear Fusion, Vol. 29, No.5 844-848 (1989)

[25] R. Paccagnella, R. Romanelli and S. Briguglio, Nuclear Fusion, Vol. 30, No. 3 545-548 (1990)

[26] M. Kotschenreuther et al., Comp. Phys. Comm. 88, 128 (1995)

[27] W. Dorland et al., Phys. Rev. Lett. 85, 5579 (2000)

[28] A. Manini et al., Nuclear Fusion, Vol. 46, No. 12 1047-1053 (2006) 
[29] F. Jenko and W. Dorland., Plasma Phy. and Control. Fusion 43, A141-A150 (2001)

[30] F. Ryter, C. Angioni, A.G. Peeters et al., Phys. Rev. Lett. 95085001 (2005)

[31] C. Angioni, Phys. Plasmas 103225 (2003)

[32] W. Suttrop et al., Plasma Phy. and Control. Fusion 42, A97-A102 (2000)

[33] G. Saibene et al., Plasma Phy. and Control. Fusion 44, 1796-1799 (2002)

[34] T.H. Osborne et al., Plasma Phy. and Control. Fusion 42, A175-A184 (2000)

[35] L.L. Lao et al., Nuclear Fusion, Vol. 41, No. 3 295-300 (2001)

[36] J. Stober et al., Plasma Phy. and Control. Fusion 42, A211-A216 (2000)

[37] T. Takizuka et al., Plasma Phy. and Control. Fusion 48, 799-806 (2006) 